\title{
Use of endocytoscopy for identification of sessile serrated adenoma/polyps and hyperplastic polyps by quantitative image analysis of the luminal areas
}

\section{(잉 $\ominus$}

\begin{abstract}
Authors
Institutions

1 Digestive Disease Center, Showa University Northern Yokohama Hospital, Yokohama, Japan

2 Digestive Disease Center, Showa University Koto Toyosu Hospital, Tokyo, Japan
\end{abstract}

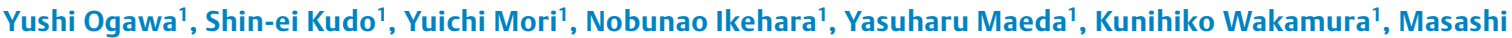
Misawa ${ }^{1}$, Toyoki Kudo ${ }^{1}$, Takemasa Hayashi ${ }^{1}$, Hideyuki Miyachi ${ }^{1}$, Atsushi Katagiri ${ }^{1}$, Fumio Ishida ${ }^{1}$, Haruhiro Inoue ${ }^{2}$

submitted 13.11 .2016

accepted after revision 22.5.2017

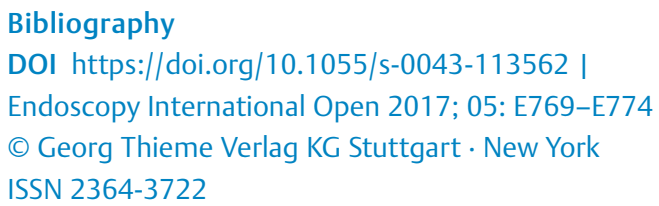

\section{ABSTRACT}

Background and study aims Recent studies that used magnifying chromoendoscopy and endocytoscopy (EC) to investigate endoscopic features of sessile serrated adenoma/polyps (SSA/Ps) suggested that a dilated crypt opening was an important indicator of SSA/Ps. However, no studies to date have measured the actual extent of dilatation. Hence, we investigated retrospectively the luminal areas using EC to determine a cutoff value for differentiating SSA/Ps from hyperplastic polyps (HPs).

Patients and methods A total of 101 lesions, including 25 SSA/Ps, $66 \mathrm{HPs}$, and 10 normal mucosal samples, assessed by an integrated-type EC were collected. For each lesion, 1 image that showed the widest lumen was selected and the average area of the contiguous 3 lumens were calculated. The cutoff value differentiating SSAPs from HPs was determined by receiver operating curve (ROC) analysis.

Results The mean luminal areas of SSA/Ps and HPs were $4152 \mu \mathrm{m}^{2}$ and $2117 \mu \mathrm{m}^{2}$, respectively. ROC analysis found that a luminal area cutoff of $3068 \mu \mathrm{m}^{2}$ had a sensitivity of $80.0 \%$, a specificity of $77.3 \%$, an accuracy of $78.0 \%$, and an area under the ROC curve of 0.865 . Furthermore, a cutoff of $\geq 556 \mu \mathrm{m}^{2}$ was found to accurately distinguish between HPs and normal mucosa (sensitivity $98.5 \%$, specificity $100 \%$, accuracy $98.7 \%$, and AUC 0.998).

Conclusions EC analysis of the luminal area is useful for differentiating between SSAPs and HPs. This approach could be adapted for computer-aided diagnosis of SSA/P.

\section{Introduction}

Historically, sporadic colorectal cancers (CRCs) were thought to arise through 1 of 2 processes: either the adenoma-carcinoma sequence or the de-novo pathway [1-3]. More recently, however, an alternative pathway of tumorigenesis, referred to as the serrated pathway, has been identified, and is implicated in up to $20 \%$ of CRCs [4].

Colorectal serrated lesions can be classified as hyperplastic polyps (HPs), sessile serrated adenoma/polyps (SSA/Ps), and traditional serrated adenomas (TSAs) [5,6].

SSA/Ps are precursors to colon cancers with microsatellite instability that should be excised, whereas HPs are reported to have a little malignant [7]. Colonoscopic identification of serrated lesions has important implications for preventing CRCs and is particularly important for differentiating SSA/Ps from HPs, which are difficult to distinguish because of their morphological similarity $[8,9]$.

To that end, several types of endoscopic imaging modalities have been evaluated, including high-resolution magnifying colonoscopy $[10,11]$, narrow-band imaging [11-13], and endocytoscopy (EC) [14]. Magnifying chromoendoscopy showed that the type II open (type II-O) pit pattern was specific to SSA/Ps, being wider and more rounded in shape owing to the dilatation of the crypts [15]. By contrast, HPs showed a type II pit pattern according to Kudo's pit classification [16]. 
On EC, SSA/Ps were shown to have oval lumens that were wide elliptical or round lumens, and small round nuclei [14]. HPs showed narrow star-shaped lumens, and small nuclei. Oval lumens are thought to correspond to dilated crypts, according to the 4th World Health Organization classification [17].

However, some lesions exhibited borderline forms that were difficult to classify as either typically "oval" or "star-like". Assessment of borderline lesions depends on the endoscopist's experience.

EC involves a contact light microscopy system integrated into the distal tip of a conventional colonoscope, and can approximate a histopathological diagnosis of the gastrointestinal tract [18-24]. EC has a $380 \times$ magnification with a focusing depth of $50 \mu \mathrm{m}$ and a field of view of $700 \times 600 \mu \mathrm{m}$. In this study, we used EC to quantitatively measure dilated crypts and to identify a dilatation cutoff value for differentiating SSA/Ps from HPs.

This study was carried out with the aim of measuring the surface area of the crypts that may provide objective evidence to confirm the diagnosis.

\section{Patients and methods}

\section{Image samples}

This retrospective study was conducted at Showa University Northern Yokohama Hospital (Yokohama, Japan) between August 2010 and December 2014. In this study, consecutive lesions with pathologically diagnosed SSA/P or HP observed on EC and resected endoscopically were analyzed. The exclusion criteria were SSA/Ps with cytological dysplasia or carcinoma, and lesions with poor visibility on EC. Moreover, we added 10 normal mucosal samples from 10 patients by biopsy.

Pathological diagnosis was carried out on the basis of the WHO criteria [17]. All of the samples were reviewed independently by a specialist gastrointestinal pathologist and a pathologist with no specialist experience in the digestive tract. If they did not agree, the diagnosis provided by the specialist gastrointestinal pathologist was used. The pathologists did not agree with the results of 3 patients in this study.

Informed consent was obtained from all patients before the procedure was performed, and the study protocol, including the use of EC, was approved by the Ethics Committee of Showa University Northern Yokohama Hospital (No. 1504-01). All participants provided written informed consent, and the study was conducted according to the Declaration of Helsinki.

\section{Image analysis}

All images were evaluated with the Image J software (National Institutes of Health, Bethesda, Maryland, United States). Luminal areas were quantified stepwise. In the first step, the data manager, who was proficient in histopathological diagnosis, downloaded complete EC images from the database server and one reviewer (Y.O.) selected 1 image that showed the widest lumen for each lesion. In the second step, 2 reviewers (Y. O., Y. M.), who were blinded to the histopathological diagnosis, manually contoured the contiguous three dilated lumens and measured their mean area with reference to the horizontal width $(700 \mu \mathrm{m})$ of the EC image. If there were more than 4 dilated crypts in the image, the 3 largest crypts were manually selected. The areas determined by the 2 reviewers were averaged. After 1 month, all images were re-measured to calculate the intraobserver agreement.

\section{Substudy of correlation between histopathologic crypts and EC lumens}

Histopathology slides were examined under a light microscope (Olympus Provis AX80) and the image was shown on a color monitor (Olympus FlexScan S1921). Slides stained with $\mathrm{H} \& \mathrm{E}$ were used as reference. We selected 37 lesions $\geq 10 \mathrm{~mm}$ (20 SSA/Ps and $17 \mathrm{HPs}$ ) and 10 normal mucosal samples. We measured 3 dilated crypts from the histopathology slide. If there were more than 4 dilated crypts, the 3 largest were manually selected by Y.O.

\section{Endocytoscopy}

All examinations were performed with an integrated-type EC scope (CF Y-0020-I, prototype from Olympus Ltd, Tokyo, Japan) [18]. This instrument has 2 observation modes: standard videoendoscopy and EC, which can be interchanged using a hand lever. EC observations are based on the principles of contact light microscopy. The EC has a 380 -fold magnification with a focusing depth of $50 \mu \mathrm{m}$ and a field of view of $700 \times 600 \mu \mathrm{m}$. EC images were obtained after staining with $1 \%$ methylene blue and $0.05 \%$ crystal violet, thus enabling clear visualization of gland duct lumens and nuclear shapes in the superficial layer that are similar to micrographic images of fixed specimens stained with hematoxylin and eosin. EC images were saved on the server that hosted the database in JPEG format with a pixel array of $640 \times 480$ in 24-bit color.

\section{Statistical analysis}

All statistical analyses were performed by using $R$ Project for Statistical Computing version 3.2.5 (Vienna, Austria). The cutoff value for differentiating SSA/Ps from HPs was evaluated by receiver operating curve (ROC) analysis, and included calculation of its sensitivity, specificity, and accuracy.

Continuous variables were compared using Student's $t$ tests, and dichotomous variables were compared using Fisher's exact tests or $X^{2}$ tests. A $P$ value $<0.05$ was considered statistically significant. To ensure reliability of diagnosis, the proportions showing agreement and the unweighted Cohen's k coefficient were determined (strengths of agreement were classified as follows: $0.01-0.2$, slight; $0.21-0.4$, fair; $0.41-0.6$, moderate; $0.61-0.8$, substantial; and $0.81-1.0$, almost perfect).

\section{Results}

\section{Image samples}

- Fig. 1 shows the analysis flow chart. A total of 101 lesions from 96 patients were screened for inclusion; 11 lesions were classified as ineligible according to the exclusion criteria (2 SSA/Ps and 4 HPs were excluded because clear EC images could not be obtained because of mucus, and 5 lesions were excluded owing to histological criteria). 21 SSA/Ps resected by EMR and 4 
32 SSA/Ps and $70 \mathrm{HPs}$ (97 patients) underwent EC between August 2010 and December 2014

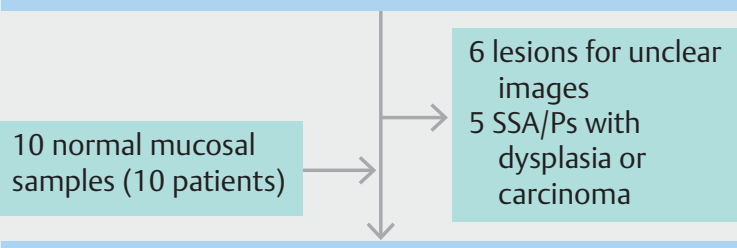

101 lesions (96 patients) analyzed

- Fig. 1 Study flow chart. EC, endocytoscopy; SSA/P, sessile serrated adenoma/polyp; HP, hyperplastic polyp

SSA/Ps by ESD. 16 HPs resected by biopsy, 7 HPs by Polypectomy and 43 HPs by EMR.

\section{Lesion characteristics}

Evaluation of the baseline characteristics of the patients with SSA/Ps and HPs revealed that the mean age and sex distributions were similar in the 2 groups ( $\triangleright$ Table 1). Evaluation of the lesions showed that the mean \pm standard deviation (SD) sizes of SSA/Ps and HPs were $18.0 \pm 10.2 \mathrm{~mm}$ and $6.9 \pm 4.8 \mathrm{~mm}$, respectively. Location in the proximal colon, a lesion size $\geq 10 \mathrm{~mm}$, and presence of a type II-O pit pattern were each significantly associated with a higher probability of diagnosing SSA/Ps.

\section{Correlation between EC luminal area and histopathology}

The mean luminal area of SSA/Ps was significantly wider than that of HPs $\left(4152 \pm 2205 \mu \mathrm{m}^{2}\right.$ vs. $2117 \pm 1167 \mu \mathrm{m}^{2}, P<0.001$; - Fig. 2a). The mean luminal area of HPs was significantly dilat-
- Table 1 Clinicopathological features of patients with sessile serrated adenoma/polyps (SSA/Ps) and hyperplastic polyps (HPs).

\begin{tabular}{|l|c|c|c|}
\hline & $\begin{array}{c}\text { SSA/Ps } \\
(\mathbf{n = 2 5 )}\end{array}$ & $\begin{array}{l}\text { HPs } \\
(\mathbf{n = 6 6 )}\end{array}$ & P value \\
\hline Mean age (y) & $58.0(12.7)$ & $62.2(11.7)$ & 0.12 \\
\hline Female & $9(36)$ & $25(38)$ & 1 \\
\hline Tumor location & & & \\
\hline - Proximal colon & $25(100)$ & $23(35)$ & $<0.001$ \\
\hline - Distal colon & 0 & $17(26)$ & 0.003 \\
\hline - Rectum & 0 & $26(39)$ & $<0.001$ \\
\hline Lesion size (mm) & $18.0(10.2)$ & $6.9(4.8)$ & $<0.001$ \\
\hline Macroscopic type & & & \\
\hline - flat & $24(96)$ & $55(83)$ & 0.17 \\
\hline - protruded & $1(4)$ & $11(17)$ & 0.17 \\
\hline Magnifying chromoendoscopy view & & \\
\hline - Type II-O & $14(69)$ & $3(5)$ & $<0.001$ \\
\hline - Type II & $11(31)$ & $60(91)$ & $<0.001$ \\
\hline
\end{tabular}

Results are expressed as mean \pm standard deviation or number of patients (\%), as appropriate.

ed, with the mean \pm SD of the luminal area being up to 6 times larger than that of the normal mucosa $\left(2117 \pm 1167 \mu \mathrm{m}^{2}\right.$ vs. $354 \pm 79 \mu \mathrm{m}^{2}, P<0.001$; Fig. $\left.2 \mathrm{a}\right)$.

ROC analysis showed that a luminal area cutoff $\geq 3068 \mu \mathrm{m}^{2}$ had a sensitivity of $80.0 \%$, a specificity of $77.3 \%$, and an accuracy of $78.0 \%$ in diagnosing SSA/Ps, with an area under the ROC curve of 0.865 . A threshold of $\geq 556 \mu \mathrm{m}^{2}$ was found to be highly

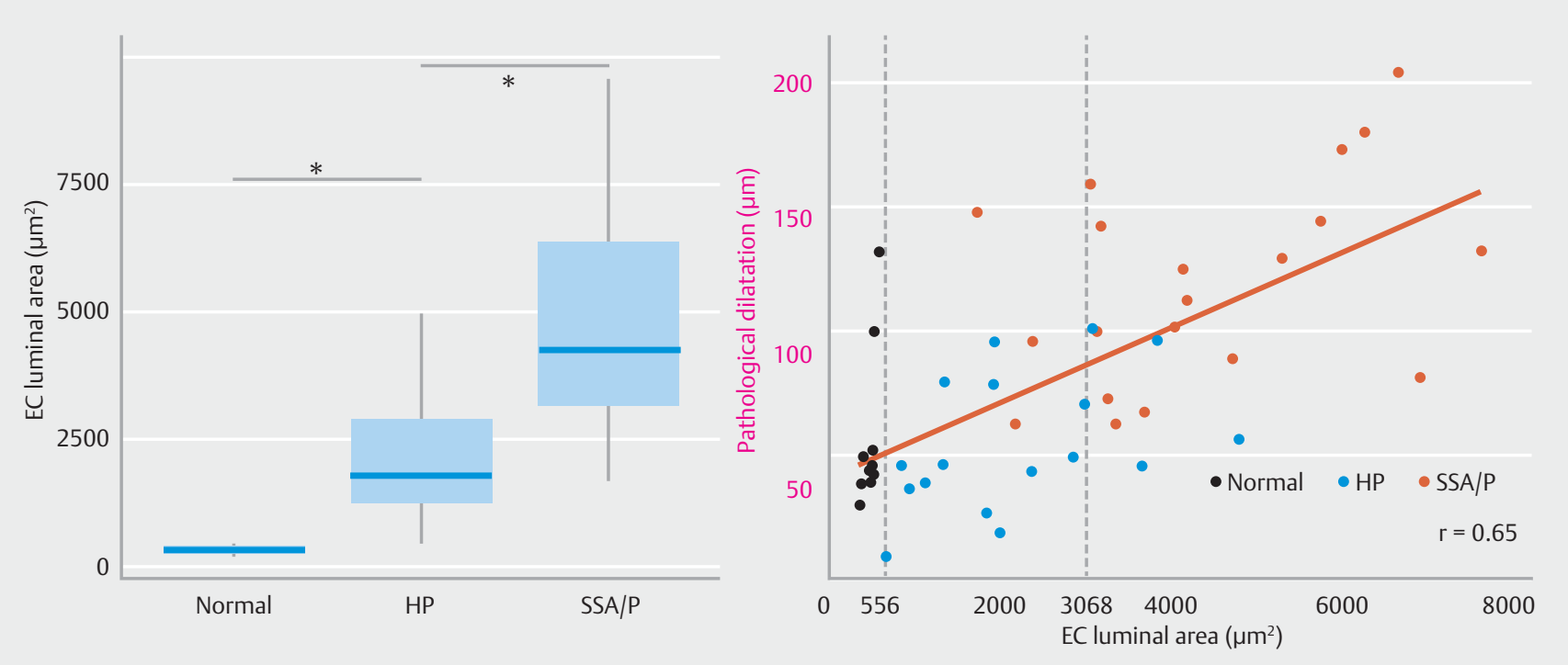

- Fig. 2 Comparison of luminal areas of normal mucosa, hyperplastic polyps (HPs), and sessile serrated adenoma/polyps (SSA/Ps). a Differences in EC luminal areas among 3 groups. ${ }^{*} P<0.001$ b Correlations between EC luminal areas and pathological crypt dilations were analyzed in 47 lesions. 

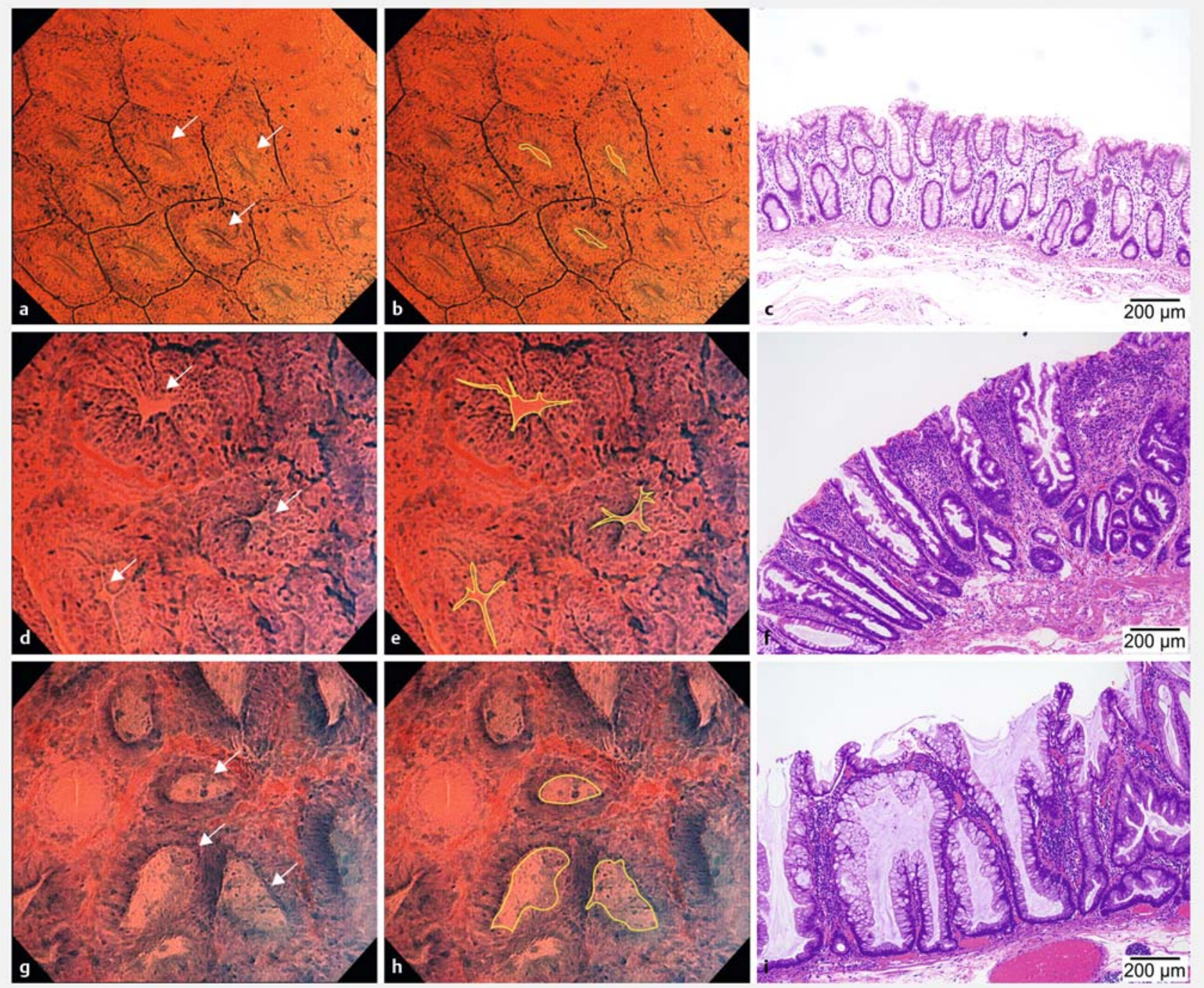

- Fig. 3 Endocytoscopy (EC) image analysis and histopathological appearance of superficial lumens of normal mucosa, hyperplastic polyp (HP), and sessile serrated adenoma/polyp (SSA/P). a Normal mucosa showed small roundish lumens under EC (white arrow). b Manual contouring of the lumens of a showed that average luminal area was $366 \mu \mathrm{m}^{2}$ (yellow line). c Histological appearance of a shows straight crypts. $\mathbf{d}$ EC image of HP showed narrow star-shaped lumens (white arrow). e Average luminal area of $\mathbf{d}$ was $1741 \mathrm{\mu m}^{2}$ (yellow line). $\mathbf{f}$ Histological appearance of $\mathbf{d}$ showed a narrowed lumen with multiple serrations. $\mathbf{g}$ EC image of SSA/P showed wide and oval lumens (white arrow). $\mathbf{h}$ Average luminal area of $G$ was $8803 \mu \mathrm{m}^{2}$ (yellow line). $\mathbf{i}$ Histological appearance of $\mathbf{g}$ showed superficial dilated crypts.

accurate in differentiating between HPs and normal mucosa, with a sensitivity of $98.5 \%$, a specificity of $100 \%$, an accuracy of $98.7 \%$, and an AUC 0.998).

Examination of the same images after 1 month showed that the mean luminal area of HPs was $2291 \mu \mathrm{m}^{2}$ and those of SSA/Ps was $4681 \mu \mathrm{m}^{2}$. There was no significant difference between the original and confirmatory measurements $(P=0.61)$. Finally, the endoscopists achieved substantial interobserver and intraobserver agreement ( $\mathrm{score}=0.71$ and 0.73 , respectively)

\section{Subgroup analysis of lesions $\geq 10 \mathrm{~mm}$}

Most SSA/Ps are $\geq 10 \mathrm{~mm}$, and we therefore carried out a subgroup analysis of lesions measuring $\geq 10 \mathrm{~mm}$. The mean area of SSA/Ps $\geq 10 \mathrm{~mm}$ was $4674 \mu \mathrm{m}^{2}$ and that of HPs was $2219 \mu \mathrm{m}^{2}$, with SSA/P lesions being significantly larger. A comparison of lesions $\geq 10 \mathrm{~mm}$ and all lesions did not reveal any difference.

\section{Substudy of correlation between histopathologic crypts and EC lumens}

We compared the luminal area as measured by EC with the dilation of surface crypt during pathological examination. These measures were found to be related (Pearson correlation coefficient $=0.65)(\triangleright$ Fig. 2 b) . 


\section{Discussion}

To our knowledge, this is the first study to investigate the actual luminal area of SSA/Ps, HPs, and normal mucosa, and we found that luminal area measurement can differentiate SSAPs from HPs. SSA/Ps may progress towards carcinoma, and since they are potential precursors of sporadic microsatellite unstable CRCs, they should be excised endoscopically. Two recent studies reported the chromoendoscopic features of SSA/Ps.

First, Kimura et al. found that a novel type II-O pit pattern was potential predictive findings of SSA/Ps compared with HPs [15]. Second, we evaluated EC features of 58 serrated lesions including $12 \mathrm{SSA} / \mathrm{Ps}$ and $27 \mathrm{HPs}$, and found that oval lumen was efficient findings of SSA/P. EC had great accuracy with a sensitivity of $83.3 \%$, and a specificity of $97.8 \%$ [14]. These dilated lumens, which are thought to correspond to histopathologically dilated crypt openings, are an important indicator of SSA/Ps [25]. Currently, however, the degree of endoscopic dilation is estimated by the endoscopist and is not measured quantitatively.

EC allows real-time celluar imaging of alimentary mucosa and provides very accurated predictions of pathology with accuracies of $94.1 \%$ [23] and $96.5 \%$ [26] for neoplastic change [20, 23, 24].

However, EC required fundamental knowledge of pathology and image training under experienced physicians to achieve accurate diagnoses and the procedure time for EC needed for washing, staining, and assessment was about 5.5 minutes [23].

On the other hand, EC enables accurate imaging by any practitioner as long as lesion contact is made. The results are objective, and the measurements do not vary depending on the technique. We think that measuring the luminal area may provide objective evidence to support diagnosis of SSA/Ps for all endoscopists including non-experts.

Our results reveal that the lumens of SSA/Ps were approximately twice as wider as those of HPs, which in turn were approximately 6 times wider than those of normal mucosa. - Fig. 3 shows the EC images, image analyses, and the histopathological appearances of normal mucosa ( $\triangleright$ Fig. 3a , $>$ Fig. 3b,

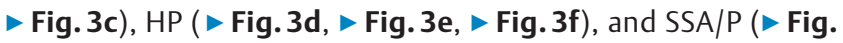
3g, $>$ Fig. 3h, $>$ Fig. 3i). The luminal size as determined by EC images corresponded to the histopathological architectures of the crypts.

In a previous study, a computer-aided diagnostic system using EC provided excellent sensitivity and accuracy for identifying neoplastic change by measuring nuclear sizes [27]. In that study, however, SSA/Ps were not evaluated because they had no nuclear abnormalities. Evaluation of the lumen was necessary for diagnosis of SSA/P. Therefore, we are developing an automated diagnosis system that can automatically recognize and measure all lumens for each image to distinguish between SSA/ Ps and HPs and identify each type.

This study had several limitations. The first limitation is selection bias introduced by selection of 3 dilated crypts from the histopathology slide, because the selection was performed manually. Second, this study was retrospective in design, and included no prospectively recorded data. It was not possible to achieve an accurate 1-to-1 correspondence between EC and pathological examination findings. Nevertheless, we normally cut out areas diagnosed by EC and tried to obtain a pathological diagnosis. In this study, we observed video recordings and excluded areas that were difficult to match to the pathological findings in an attempt to pair as many lesions as possible.

Finally, the major limitation was measurement bias due to obtaining these measurements manually. Moreover, only 3 lumens were measured per image in this study. To evaluate more objectively, computer-aided analysis is considered favorable as a next challenge

\section{Conclusion}

In conclusion, this study showed that EC is a promising diagnostic tool for distinguishing between SSA/Ps and HPs. This approach may be incorporated into computer-aided diagnosis of SSA/Ps in the future.

\section{Acknowledgements}

The authors thank Drs. Makoto Kutsukawa, Akihiro Yamauchi, and Kenta Igarashi, Digestive Disease Center, Showa University Northern Yokohama Hospital, Yokohama, Japan, and Olympus Co, Ltd. for their invaluable support for the study. We also thank Editage (www.editage.jp) for English language editing.

\section{Competing interests}

None

References

[1] Kudo $S$, Tamura $S$, Hirota $S$ et al. The problem of de novo colorectal carcinoma. Eur J Cancer 1995; 31A: $1118-1120$

[2] Morson BC. Precancerous lesions of the colon and rectum. Classification and controversial issues. JAMA 1962; 179: $316-321$

[3] Vogelstein B, Fearon ER, Hamilton SR et al. Genetic alterations during colorectal-tumor development. N Engl J Med 1988; 319: 525-532

[4] Huang CS, O'Brien MJ, Yang S et al. Hyperplastic polyps, serrated adenomas, and the serrated polyp neoplasia pathway. Am J Gastroenterol 2004; 99: $2242-2255$

[5] Snover DC, Jass JR, Fenoglio-Preiser C et al. Serrated Polyps of the Large Intestine: A Morphologic and Molecular Review of an Evolving Concept. Am J Clin Pathol 2005; 124: 380 - 391

[6] Leggett B, Whitehall V. Role of the serrated pathway in colorectal cancer pathogenesis. Gastroenterology 2010; 138: 2088-2100

[7] East JE, Vieth M, Rex DK. Serrated lesions in colorectal cancer screening: detection, resection, pathology and surveillance. Gut 2015; 64 : $991-1000$

[8] Kashida H, Ikehara N, Hamatani S et al. Endoscopic characteristics of colorectal serrated lesions. Hepatogastroenterology 2011; 58: $1163-1167$

[9] Hetzel JT, Huang CS, Coukos JA et al. Variation in the detection of serrated polyps in an average risk colorectal cancer screening cohort. Am J Gastroenterol 2010; 105: 2656-2664 
[10] Kumar S, Fioritto A, Mitani A et al. Optical biopsy of sessile serrated adenomas: do these lesions resemble hyperplastic polyps under narrow-band imaging? Gastrointest Endosc 2013; 78: 902 - 909

[11] Hazewinkel Y, Lopez-Ceron M, East JE et al. Endoscopic features of sessile serrated adenomas: validation by international experts using high-resolution white-light endoscopy and narrow-band imaging. Gastrointest Endosc 2013; 77: 916-924

[12] Uraoka T, Higashi R, Horii J et al. Prospective evaluation of endoscopic criteria characteristic of sessile serrated adenomas/polyps. J Gastroenterol 2014; 50: 555-563

[13] Yamashina T, Takeuchi Y, Uedo N et al. Diagnostic features of sessile serrated adenoma/polyps on magnifying narrow band imaging: $\mathrm{A}$ prospective study of diagnostic accuracy. J Gastroenterol Hepatol 2015; 30: 117-123

[14] Kutsukawa M, Kudo SE, Ikehara $\mathrm{N}$ et al. Efficiency of endocytoscopy in differentiating types of serrated polyps. Gastrointest Endosc 2014; 79: $648-656$

[15] Kimura T, Yamamoto E, Yamano HO et al. A novel pit pattern identifies the precursor of colorectal cancer derived from sessile serrated adenoma. Am J Gastroenterol 2012; 107: 460-469

[16] Kudo S, Rubio CA, Teixeira CR et al. Pit pattern in colorectal neoplasia: endoscopic magnifying view. Endoscopy 2001; 33: 367-373

[17] Snover DC, Ahnen DJ, Burt RW et al. Serrated polyps of the colon and rectum and serrated polyposis. In: Bosman FT, Carneiro F, Hruban RH, Theise ND (eds) WHO Classification of Tumours of the Digestive System. Lyon, France: IARC Press; 2010: 160-165

[18] Inoue H, Sasajima K, Kaga M et al. Endoscopic in vivo evaluation of tissue atypia in the esophagus using a newly designed integrated endocytoscope: a pilot trial. Endoscopy 2006; 38: 891 - 895
[19] Kaise M, Ohkura Y, lizuka T et al. Endocytoscopy is a promising modality with high diagnostic accuracy for gastric cancer. Endoscopy 2015; 47: 19-25

[20] Kudo SE, Wakamura K, Ikehara $\mathrm{N}$ et al. Diagnosis of colorectal lesions with a novel endocytoscopic classification - a pilot study. Endoscopy 2011; 43: 869-875

[21] Neumann H, Kudo SE, Kiesslich R et al. Advanced colonoscopic imaging using endocytoscopy. Dig Endosc 2015; 27: 232 - 238

[22] Sasajima K, Kudo SE, Inoue $\mathrm{H}$ et al. Real-time in vivo virtual histology of colorectal lesions when using the endocytoscopy system. Gastrointest Endosc 2006; 63: 1010 - 1017

[23] Mori Y, Kudo S, Ikehara N et al. Comprehensive diagnostic ability of endocytoscopy compared with biopsy for colorectal neoplasms: a prospective randomized noninferiority trial. Endoscopy 2013; 45: $98-105$

[24] Kudo SE, Mori Y, Wakamura K et al. Endocytoscopy can provide additional diagnostic ability to magnifying chromoendoscopy for colorectal neoplasms. J Gastroenterol Hepatol 2014; 29: 83 -90

[25] Rex DK, Ahnen DJ, Baron JA et al. Serrated lesions of the colorectum: review and recommendations from an expert panel. Am J Gastroenterol 2012; 107: 1315-1329

[26] Neumann H, Fuchs FS, Vieth M et al. Review article: in vivo imaging by endocytoscopy. Aliment Pharmacol Ther 2011; 33: 1183-1193

[27] Mori Y, Kudo SE, Wakamura K et al. Novel computer-aided diagnostic system for colorectal lesions by using endocytoscopy (with videos). Gastrointest Endosc 2015; 81: 621-629 